\title{
High-repetition rate acoustic-induced Q-switched all-fiber laser
}

\author{
D. Zalvidea ${ }^{\text {a }}$, N.A. Russo ${ }^{\text {a }}$, R. Duchowicz ${ }^{\text {a,b }}$, M. Delgado-Pinar ${ }^{c}$, A. Díez ${ }^{\text {c,* }}$, \\ J.L. Cruz ${ }^{\text {c }}$ M.V. Andrés ${ }^{\mathrm{c}}$ \\ a Centro de Investigaciones Opticas, CIOp (CONICET, CIC), La Plata, Argentina \\ b Departamento de Física, Fac. Ciencias Exactas, UNLP, La Plata, Argentina \\ c Departamento de Física Aplicada, ICMUV, Universidad de Valencia, Dr. Moliner 50, 46100 Burjassot, Spain
}

Received 20 May 2004; received in revised form 9 September 2004; accepted 10 September 2004

\begin{abstract}
We report a high-repetition rate actively Q-switched all-fiber laser. The acousto-optic interaction controls the cou= pling between co-propagating core and cladding modes and is used to modulate the optical losses of the cavity, which permits to perform active Q-switching. Using $1.4 \mathrm{~m}$ of $300 \mathrm{ppm}$ Er-doped fiber and a maximum pump power of 120 $\mathrm{mW}$, we have obtained up to $1 \mathrm{~W}$ peak power pulses, with a pulse repetition rate that can be continuously varied from $1 \mathrm{~Hz}$ to $120 \mathrm{kHz}$ and a pulse width that changes from $70 \mathrm{~ns}$ to $2.2 \mu \mathrm{s}$.

(c) 2004 Elsevier B.V. All rights reserved.
\end{abstract}

\section{Introduction}

The development of new fiber optic laser systems is of permanent interest in the optical field, especially on the communication spectral window. In particular, erbium-doped fiber lasers have shown a variety of potential applications, such as sources for WDM and soliton communications

\footnotetext{
* Corresponding author. Tel.: 34 963983431; fax: 34 963983146.

E-mail address: antonio.diez@uv.es (A. Díez).
}

systems, medicine, sensing and spectroscopy. In order to produce an erbium-doped Q-switched laser many different approaches can be employed. Passive Q-switching can be obtained by using a saturable absorber constructed by inserting a segment of samarium-doped fiber into a ring cavity [1]. Active Q-switching was performed using an electro-optic modulator, an acousto-optic modulator (AOM), or an intensity modulator based in the transmission of a coupler in the cavity [2-4].

On the other hand, U-V written fiber Bragg gratings (FBGs) as a reliable fiber technology has 
generated very important advances in the development of fiber-based devices and systems. In particular, FBGs can be used as all-fiber mirrors to define a Fabry-Perot cavity. The laser emission wavelength is, in this case, determined by the Bragg wavelengths of the fiber gratings. Many designs of fiber lasers for optical communications, making use of fiber gratings to the resonant cavity, have been previously reported [5].

In a previous work, we demonstrated active Qswitching of an erbium-doped fiber laser that employs a pair of fiber Bragg gratings as reflective mirrors. The laser operation was based on modulating the Bragg wavelength of one grating fixed to a piezoelectric ceramic tube. By using this very simple scheme, we obtained a Q-switched laser in the erbium spectral gain region with a high laser efficiency of energy conversion. Pumping at 76 $\mathrm{mW}$ and operating the laser at $18.5 \mathrm{kHz}$, an efficiency of $26 \%$ was achieved [6,7].

Recently, Liu and co-workers [8] demonstrated an actively Q-switched fiber laser in which an acousto-optic-based fiber attenuator $[9,10]$ placed inside the cavity was employed to actively control the cavity losses. Microbending induced in the fiber by a flexural acoustic wave produces resonant coupling between the fundamental core mode and cladding modes, which are rapidly attenuated by the fiber coating [11]. Pulses of several $\mu \mathrm{J}$ per pulse, 150 ns wide and a repetition rate of up to $5 \mathrm{kHz}$ was reported.

In this paper, we report an optimized design of a Q-switched all-fiber laser based on the idea reported by Liu and co-workers [8]. We have included two modifications to Liu's design to improve the performance of the system. First, we used photosensitive erbium-doped fiber, so that the FBG used as reflective elements were written in the active fiber itself, giving rise to a splice-less laser cavity. Second, to enhance the efficiency of the acousto-optic attenuator, it was made on a section of fiber that was tapered down using a fusion-and-pulling technique. This allows making acousto-optic attenuators as efficient as the one used in [8], which was made using HF etching, but with smaller reductions of the fiber diameter. This is relevant to the performance of the Q-switched laser, i.e. the maximum repetition rate achievable, since the time response of the attenuator shortens as the fiber diameter increases due to the dependence of the acoustic velocity with the diameter [11].

We demonstrate a Q-switched laser with up to 1 $\mathrm{W}$ peak power, pulses from $70 \mathrm{~ns}$ to $2.2 \mu$ s wide, and a pulse repetition rate that can be continuously varied from $1 \mathrm{~Hz}$ to $120 \mathrm{kHz}$.

\section{Experimental setup and results}

Fig. 1 shows a schematic diagram of the Qswitched erbium-doped fiber laser. The fiber used in the experiments was an erbium-doped germanosilicate fiber containing $300 \mathrm{ppm} \mathrm{Er}^{3+}$, with a cut-off wavelength of $965 \mathrm{~nm}$, and a numerical aperture (NA) of 0.23 . Two Bragg gratings, FBG1 and FBG2, were written on the core of the $\mathrm{Er}^{3+}$-doped fiber by UV exposure using a doubled argon laser and a uniform period phase mask, to define a cavity of $140 \mathrm{~cm}$ in length. The fiber was hydrogen loaded to enhance its photosensitivity. The Bragg wavelength of both FBGs was $1546.6 \mathrm{~nm}$. The reflectivity of FBG1 and FBG2 was $60 \%$ and $99 \%$, respectively, and the bandwidth was 0.15 and $0.25 \mathrm{~nm}$.

The acousto-optic attenuator was implemented on the $\mathrm{Er}^{3+}$-doped fiber itself. Flexural acoustic waves were imposed on the fiber using a piezoelec-

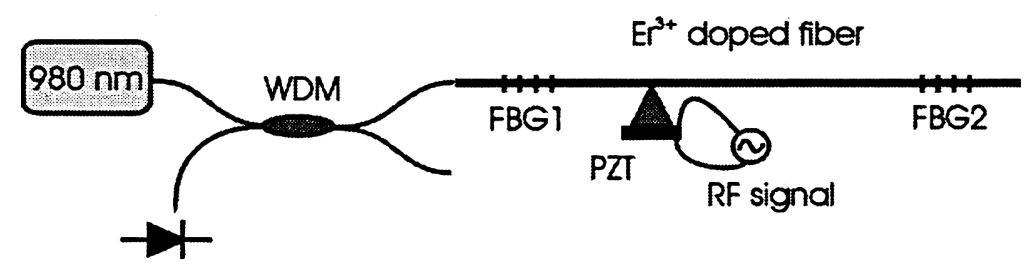

Fig. 1. Experimental setup. 
tric transducer (a PZT ceramic disc of $1 \mathrm{~cm}$ diameter) driven by a RF signal, and an aluminum horn (see Fig. 1). The acousto-optic interaction takes place in a section of $8 \mathrm{~cm}$ long of the $\mathrm{Er}^{3+}$ fiber. To enhance the acousto-optic interaction, this section of fiber was tapered down from 125 to $80 \mu \mathrm{m}$ in diameter using a standard fusion-and-pulling tapering technique [12]. We found that resonant coupling between the fundamental core mode $\mathrm{LP}_{01}$ and the $\mathrm{LP}_{12}$ cladding mode occurred at around $1.55 \mu \mathrm{m}$ when the frequency of the RF signal was $1.2 \mathrm{MHz}$. Fig. 2 shows an example of the transmission spectrum of the fiber when an RF signal of $1.201 \mathrm{MHz}$ and an amplitude of $15 \mathrm{~V}$ was applied to the piezoelectric. An attenuation peak centered at $1546.6 \mathrm{~nm}$ (i.e., the Bragg wavelength of the FBGs) as deep as $16 \mathrm{~dB}$ is observed. The inset of Fig. 2 shows the tunability of the dip with the frequency of the RF signal. Coupling from the $\mathrm{LP}_{01}$ to other cladding modes was also observed for other frequencies. Measurements shown in Fig. 2 were done before the FBGs were written.

Fig. 3 shows the time response of the acoustooptic attenuator. For this experiment, the fiber was illuminated using a laser diode tuned at the acousto-optic resonant wavelength and the FBGs were detuned to avoid reflections from them. Fig. 3(a) shows the electrical signal applied to the piezoelectric transducer and Fig. 3(b) shows the cor-

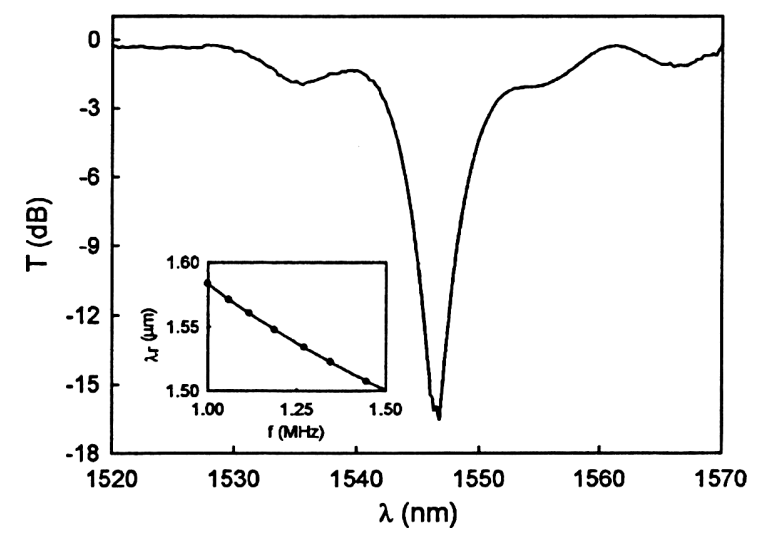

Fig. 2. Transmission spectrum when an electrical signal of $15 \mathrm{~V}$ and 1.201 MHz is applied to the piezoelectric. The dip is caused by the resonant coupling between the $\mathrm{LP}_{01}$ core mode and the $\mathrm{LP}_{12}$ cladding mode. Inset: acousto-optic resonant wavelength $\lambda_{\mathrm{r}}$ as a function of the RF frequency.
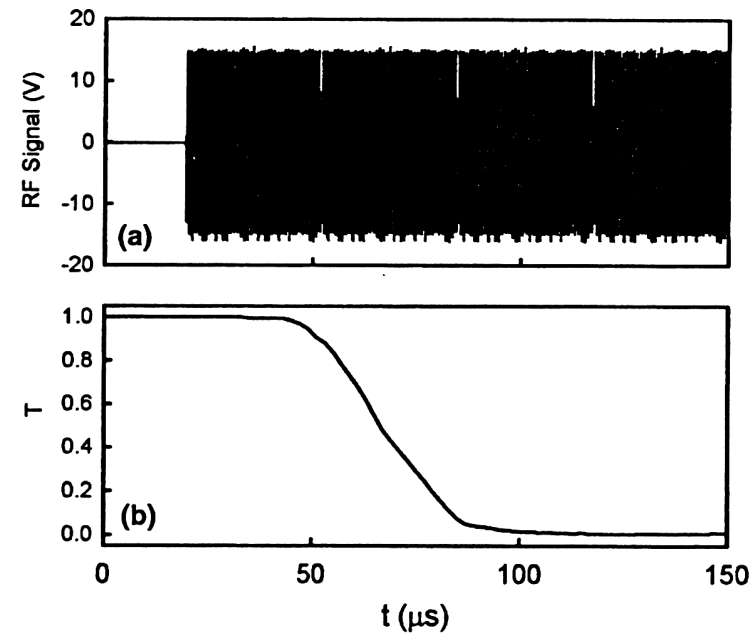

Fig. 3. Time response of the acousto-optic process. (a) Electrical signal applied to the piezoelectric and (b) light transmission.

responding optical transmission. The switching time, i.e., the time that the signal takes to change from $100 \%$ to $0 \%$, is about $50 \mu$ s. This value is an order of magnitude shorter than the best value reported in [8]. A delay of $30 \mu$ s between the electrical and the optical signals is also observed, which is caused by the overall system that generates the acoustic wave.

The active medium was pumped through a WDM coupler using a pigtailed semiconductor laser diode $(980 \mathrm{~nm}, 125 \mathrm{~mW}$ of maximum optical power). The optical output from FBG1 was detected using a $125 \mathrm{MHz}$ bandwidth InGaAs photodetector. The efficiency of the fiber laser in CW operation (without acoustic excitation) was about $5 \%$ and the threshold was $4 \mathrm{~mW}$.

To perform Q-switching, the RF signal was amplitude modulated by a rectangular wave of variable frequency and duty cycle. This modulation produced on-off periods of the acoustic wave being imposed to the fiber, which results in a modulation of the cavity losses at the laser emission wavelength.

Fig. 4 shows an example of the laser system running at $10 \mathrm{kHz}$. Fig. 4(a) is the laser signal and Fig. 4(b) shows the modulation signal. The duty cycle was adjusted to optimize the amplitude and the stability of the pulses. Fig. 4(c) shows the performance of the fiber attenuator under the same 

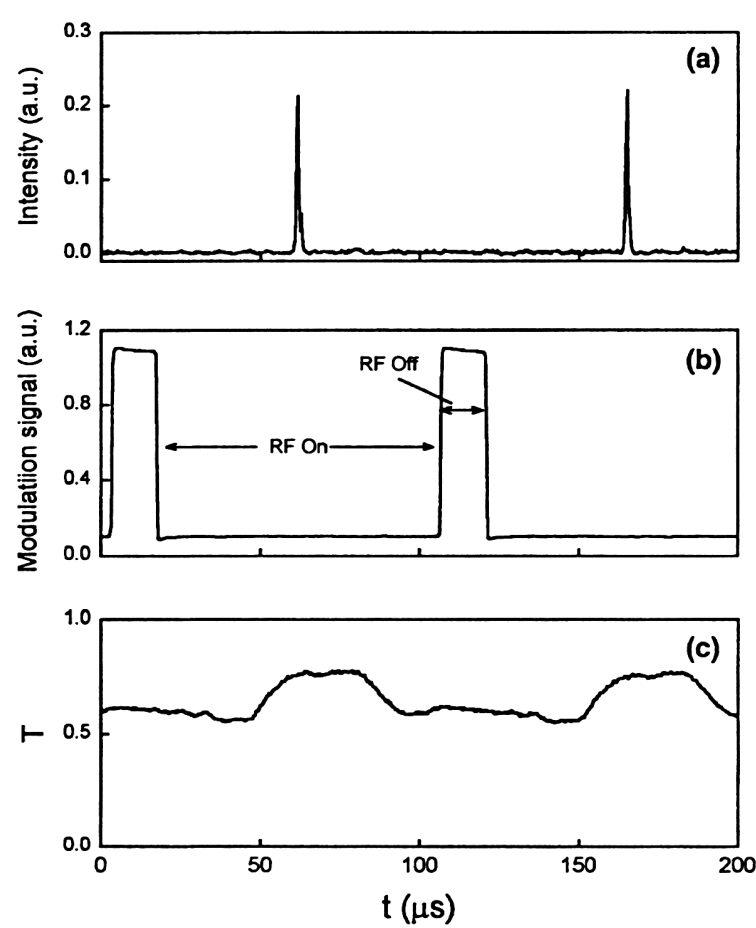

Fig. 4. (a) Laser output when operating at $10 \mathrm{kHz}$. (b) Corresponding modulation signal. (c) Light transmission under the same piezoelectric excitation.

piezoelectric excitation. This measurement was done in a similar way as in Fig. 3 (with no pump and detuning the FBGs to avoid reflections), and it shows that just a few percent of attenuation change is enough to generate Q-switching. Fig. 5

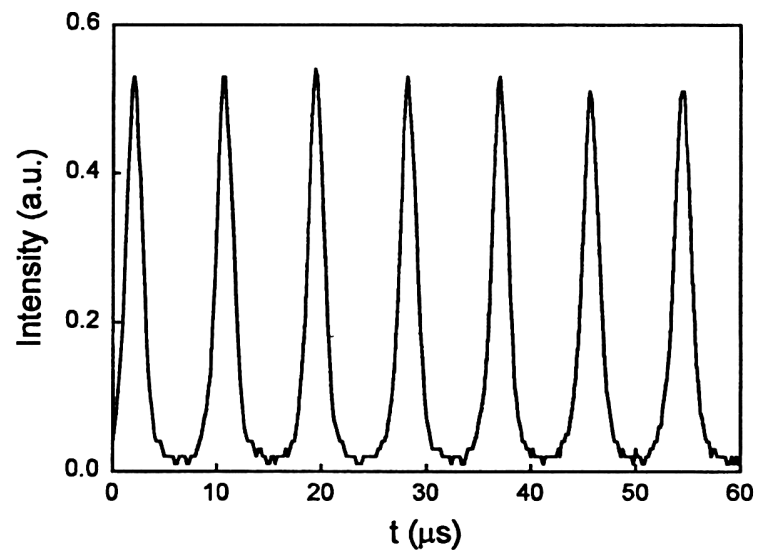

Fig. 5. Laser output when operating at $120 \mathrm{kHz}$. shows an example of the laser operating at 120 $\mathrm{kHz}$. Higher repetition rates could be attainable by reducing the time response of the acousto-optic attenuator. This could be achieved, for example, by shortening the length of the acousto-optic attenuator, provided that enough acoustic power is available to obtain the required attenuation level to perform Q-switching.

Fig. 6(a) gives the output peak power of the Qswitched laser pulses against the continuous pump power, for several repetition frequencies. Pulses of up to $1 \mathrm{~W}$ were obtained at low frequencies. Pulseto-pulse fluctuation of the peak power was typically about 5\%. Fig. 6(b) gives the peak power and the pulse width as a function of the repetition rate, when pumping at $85 \mathrm{~mW}$. Around $1-2 \mathrm{kHz}$ the peak power starts to decrease significantly and the pulse width to increase, as expected for a medium with an upper-level effective lifetime of 1 ms. Pulses from $70 \mathrm{~ns}$ to $2.2 \mu$ s wide were obtained. The long microsecond pulses at high-repetition rates may limit the use of the system in some applications. Shorter pulses could be obtained by reducing the length of the laser cavity and also by improving the time response of the acoustooptic modulator.
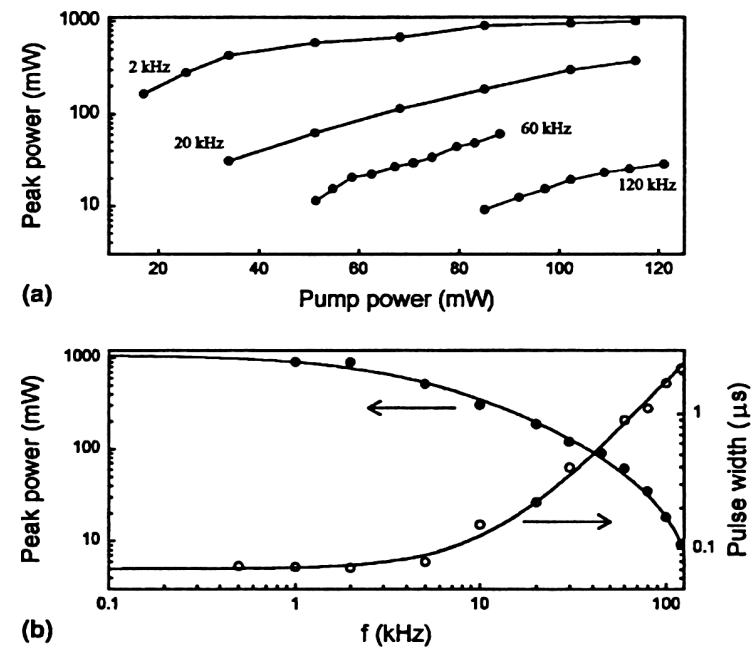

Fig. 6. (a) Optical peak power versus continuous pump power, for several pulse repetition frequencies. (b) Optical peak power and pulse width against pulse repetition frequency when pumping at $85 \mathrm{~mW}$. 


\section{Summary}

In summary, we have reported a high-repetition rate acoustic-induced Q-switched all-fiber laser. A single piece of $\mathrm{Er}^{3+}$-doped germanosilicate fiber has been employed to make the laser, writing the FBGs and performing the modulation of the cavity losses in the fiber itself. Q-switching was performed by controlling the optical losses in the $\mathrm{Er}^{3+}$ fiber by coupling light from the core mode to cladding modes using flexural acoustic waves. The active Q-switching can be operated continuously from $1 \mathrm{~Hz}$ to $120 \mathrm{kHz}$ and the pulse width changes from $70 \mathrm{~ns}$ to $2.2 \mu \mathrm{s}$. Using $1.4 \mathrm{~m}$ of 300 ppm Er-doped fiber and pumping powers lower than $120 \mathrm{~mW}$, up to $1 \mathrm{~W}$ peak power pulses were achieved at $2 \mathrm{kHz}$ repetition rate.

\section{Acknowledgments}

This work was financially supported by the Ministerio de Ciencia y Tecnología of Spain (Grant TIC2002-04527-C02-01), NKT Research \& Innovation and the Generalitat Valenciana (grupos03/227). R. Duchowicz, N.A. Russo and D. Zalvidea acknowledge financial support from the Consejo Nacional de Investigaciones Cientifi- cas y Técnicas (CONICET), the Facultad de Ciencias Exactas, Universidad Nacional de La Plata and the Comisión de Investigaciones Científicas de la Prov. de Buenos Aires (CIC), Argentina.

\section{References}

[1] L. Luo, P.L. Chu, Opt. Commun. 161 (1999) 257.

[2] H.H. Kee, G.P. Lees, T.P. Newson, Electron. Lett. 34 (1998) 1318.

[3] P. Roy, D. Pagnoux, L. Mouneu, T. Midavaine, Electron. Lett. 33 (1997) 1317.

[4] A. Chandonnet, G. Larose, Opt. Eng. 32 (1993) 2031.

[5] V. Mizrahi, D.J. DiGiovanni, R.M. Atkins, S.G. Grubb, Y.K. Park, M.P. Delavaux, J. Lightwave Technol. 11 (1993) 2021.

[6] N.A. Russo, R. Duchowicz, J. Mora, J.L. Cruz, M.V. Andrés, Opt. Commun. 210 (2002) 361.

[7] R. Duchowicz, N.A. Russo, E. Sicre, M.V. Andres, J. Opt. A 5 (2003) 5216.

[8] D.W. Huang, W.F. Liu, C.C. Yang, IEEE Photon. Technol. Lett. 12 (2000) 1153.

[9] D.W. Huang, W.F. Liu, C.W. Wu, C.C. Yang, IEEE Photon. Technol. Lett. 12 (2000) 176.

[10] H.S. Kim, S.H. Yun, I.K. Kwang, B.Y. Kim, Opt. Lett. 22 (1997) 1476.

[11] T.A. Birks, P.St.J. Russell, D.O. Culverhouse, J. Lightwave Technol. 14 (1996) 2519.

[12] R.P. Kenny, T.A. Birks, K.P. Oakley, Electron. Lett. 77 (1991) 1654. 